\title{
A Multisensory Virtual Environment for OSH Training
}

\author{
Mina Tahsiri \\ University of Nottingham
}

\author{
Glyn Lawson* \\ University of Nottingham
}

\author{
Che Abdullah \\ University of Nottingham
}

\author{
Tessa Roper \\ University of Nottingham
}

\begin{abstract}
This paper presents a multisensory and low-cost virtual training simulator developed in Unity $3 \mathrm{D}$, with the aim of improving the effectiveness of Occupational Safety and Health (OSH) training. The prototype system facilitates heat and smell feedback functions operated by an Arduino microprocessor and triggered based on the proximity of the avatar to receptive 4 within the Virtual Environment (VE). The prototype enables the creation of bespoke virtual representations using the $3 \mathrm{D}$ scanning function of the Google Tango device making multisensory VE OSH training a feasible and versatile approach in the short-term future.
\end{abstract}

Keywords: Multisensory, Virtual Environment, training, safety.

Index Terms: • Human-centered computing Virtual reality - Applied computing Interactive learning environments $\bullet$ Software and its engineering $\sim$ Virtual worlds training simulations

\section{INTRODUCTION}

In safety-critical contexts it is not usually practical, cost-effective or ethical to conduct training in the real situation. Virtual Environment (VE)-based training offers a promising alternative. It has been seen to improve knowledge uptake during training of pre-shift inspection of haulage trucks and safety and operational procedures for conveyor systems in the mining industry [1] and ensure competence before exposure to high-risk scenarios [3].

However, Virtual Environments are not always adequate for effective OSH training. For example, Smith and Trenholme [2] found that participants using a virtual fire drill environment demonstrated unsafe behaviours such as opening doors with smoke coming from underneath them, attributed to a lack of heat in their simulation. Multimodal feedback may improve validity and effectiveness in these cases, but the effectiveness of multimodal VEs has not yet been tested in OSH training.

The VE prototype presented in this paper is aimed for use in companies in which identification of and dealing with hazards such as fire, smoke, chemical and gas leakage are key to the OSH training process. The aim in this research project is to test a system comprising low-cost existing technologies, to see if a configuration of affordable hardware is effective in OSH training.

\section{Virtual enVironment}

The VE is built by scanning the premises in which OSH training is to take place using Google Tango which allows for an easy and quick capture of a 3D point cloud (Figure 1). This provides an effective way for a company with limited development expertise or resource to replicate their own premises, enhancing the relevance of training and potentially improving knowledge transfer to real situations.

\footnotetext{
*glyn.lawson@nottingham.ac.uk
}

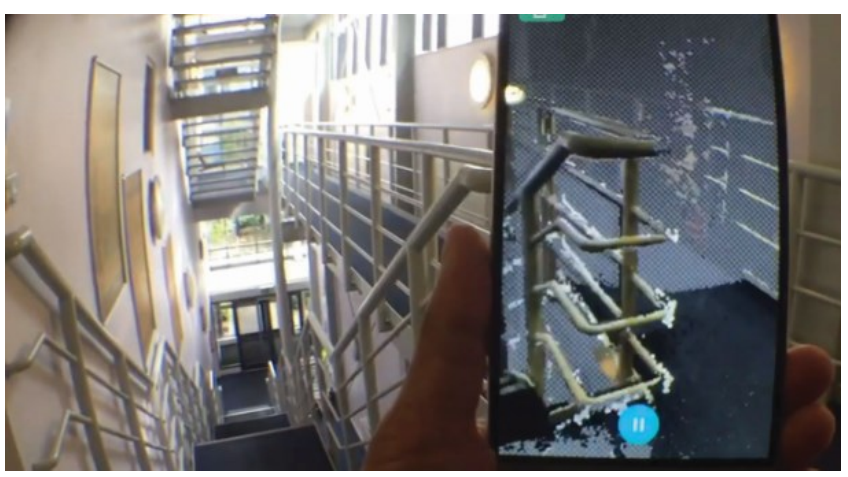

Figure 1: Using the Google Tango scanner to capture a 3D point cloud of the premises.

Using MeshLab, the collected data are converted into a mesh, used in Unity 3D. The user can navigate an avatar around the VE with a first-person perspective. Two types of colliders are implemented (Figure 2). The first type of colliders function as barriers for the movement of the avatar, for example located behind the mesh representing a wall. The second type of colliders are placed where the proximity of the avatar to an element activates a command, either triggering a virtual activity like sounding an alarm or opening an emergency door, or triggering a physical component such as the heaters and scent diffusers.

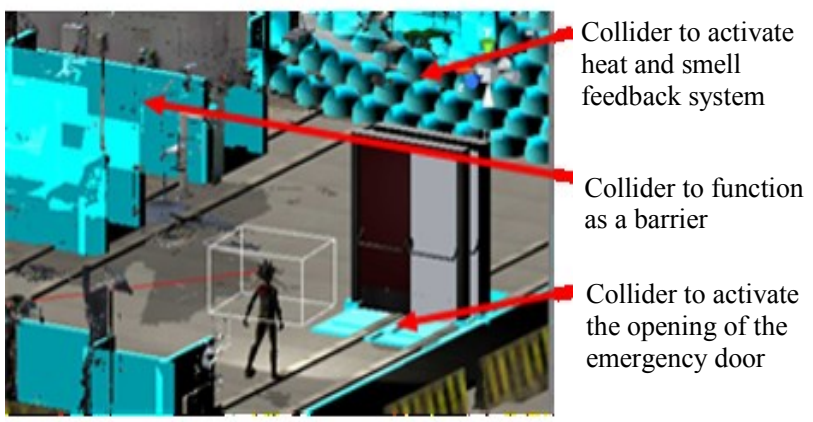

Figure 2: Different types of colliders designed with the Unity VE.

The VE enables the training supervisor to configure the training scenarios by changing the starting location of the avatar and the location of colliders and hazards. The VE allows the trainer to provide concurrent instructions to the trainee, in addition to retrospective feedback upon completion of the training, which can include playback and alternative views of the VE to allow the trainee to explore the consequences of their actions.

\section{FeedBack AND CONTROL SYSTEM}

A control system using Arduino has been designed for the heat and scent simulations (Figure 3). The Arduino hardware is managed by a Python control software synchronised with the Unity VE. Studies evaluating the heat and scent systems are in process with the aim of optimising the trainee's sensory experience. 


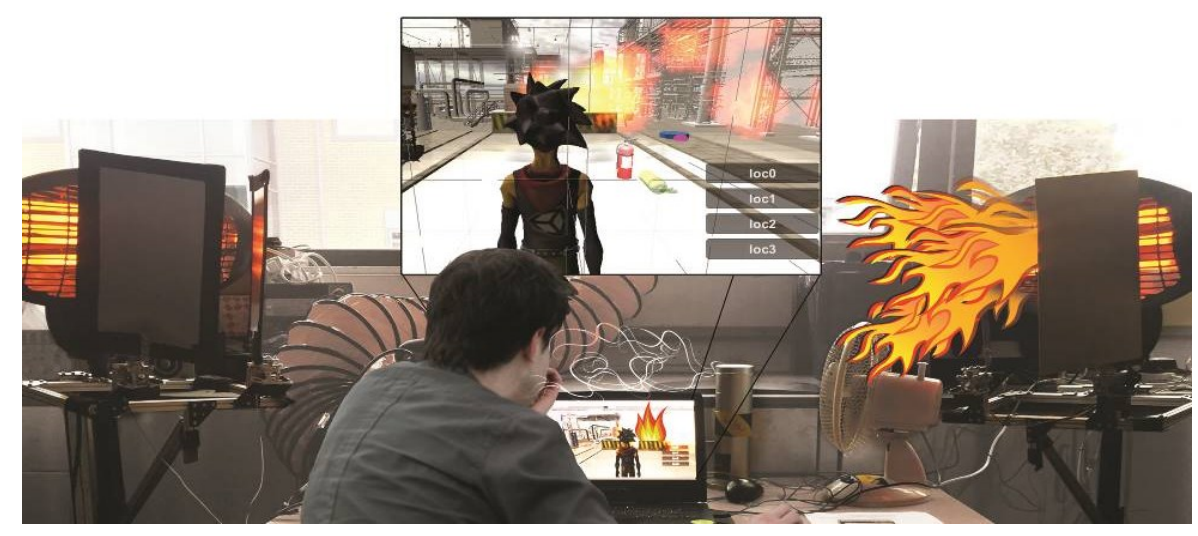

Figure 3: The set-up of the multisensory VE prototype, comprising two heaters, scent diffuser, fan and extractor.

\subsection{Heating System}

In the prototype system, two low-cost $2 \mathrm{KW}$ infrared heating devices are used to deliver heat, placed to the left and right in front of the user such that both can be activated depending on the position of the avatar relative to the heat source in the VE. Each heater is shielded by two metal sheets or "fins" which are servocontrolled and open like shutters as the user approaches the heat source, closing again when they move away. This fin system was introduced to overcome time-lag of the heaters warming up as the avatar approaches the heat source in the VE. A radial zoning centred on each hazardous element is used for the allocation of the colliders (Figure 4). As the avatar moves towards the centre of the hazard, the fins open more and the trainee experiences more heat. The optimal location for the two heaters is under evaluation. Measures such as the time it takes for participants to react to the heaters, perceived realism of the heat, and accuracy in identifying the direction of the heat source whilst engaged in the virtual environment are being investigated.

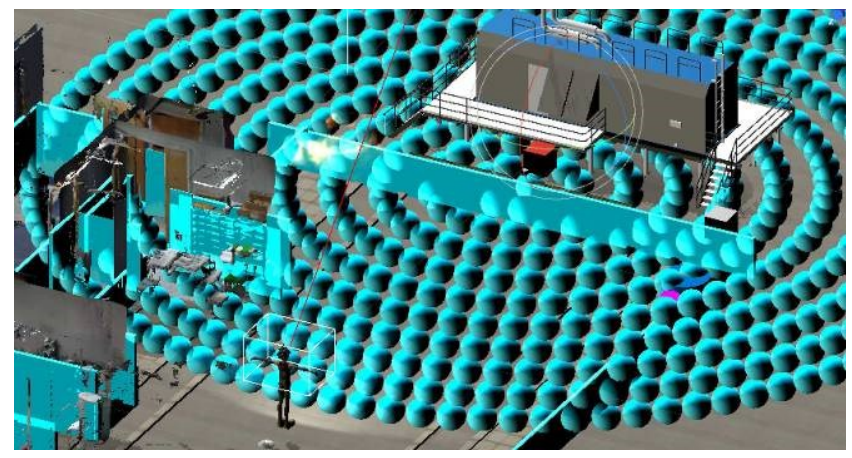

Figure 4: Radial zoning of colliders as triggers for the heaters.

\subsection{Scent Diffusing System}

A scent diffuser device placed near the trainee contains fragrances relevant to the scenario such as smoke, burning material or chemicals. The diffuser emits a small amount of fragrance in the direction of the trainee. A fan in front of the user and a small extractor unit behind them help control the direction of the scent while containing it within the test area and extracting it safely.

\subsection{Classifier Development}

The system will include "Classifer" objects with predefined behaviours that can be used as part of training scenarios, such as alarms that can be activated by the user. The aim is that any VE can be customised with elements related to the training objective. Classifier development is based on two approaches: 1) userinsertion of classifier objects into the scene, and 2) automatic determination of important elements to a scenario design, such as 'emergency signage', using a 2D pattern-match algorithm to automatically classify.

\section{Next Steps}

The prototype has been shown to a number of users and stakeholders, receiving positive feedback, and is currently undergoing refinement. In particular we want to ensure feasibility of use by the bodies interested in incorporating the system into their OSH training, with the aim of reducing costs of creating the $\mathrm{VE}$ and minimising prerequisite expertise. We are developing instructions for premises scanning that will minimise the amount of noise generated in the point cloud. We are also developing a strategy for building a mesh to be used in Unity 3D that reduces file size, and allows remodelling of the premises within the VE. We are refining the realism of the VE experience, which will be informed by the in-progress user tests investigating the effects of hardware positioning.

The final prototype will be tested alongside unimodal training and traditional approaches in user studies. The effectiveness of the training, the validity of behaviours in the environment, and participants' subjective responses to the training (such as motivation and engagement) will be compared.

\section{ACKNOWLEDGMENTS}

This work is supported by the Institution of Occupational Safety and Health through its research fund.

\section{REFERENCES}

[1] R. McMahan, S. Schafrik, D. Bowman and M. Karmis. Virtual environments for surface mining powered haulage training. In J. Brune, editor, Proceedings of SME Symposium Celebrating 100 Years of Mining Research (Phoenix, Arizona, 2009) Extracting the science: a century of mining research, The Society for Mining, Metallurgy and Exploration, pages 520-528, 2010.

[2] S. Smith and D. Trenholme. Rapid prototyping in virtual fire drill environment using computer game technology. Fire Safety Journal, volume 44, issue 4, pages 559-569, 2009.

[3] R. Webber-Youngman and E. Van Wyk. Incident reconstruction simulations-potential impact on the prevention of the future mine incidents. Journal of the Southern African Institute of Mining and Metallurgy, volume 113, issue 6, pages 519-528, 2013. 